\title{
Dual-Homing Based Protection for Enhanced Network Availability and Resource Efficiency
}

\author{
S. Abeywickrama ${ }^{1}$, M. Furdek ${ }^{2,3}$, P. Monti ${ }^{2}$, L. Wosinska ${ }^{2}$, A. Nag ${ }^{4}$, E Wong ${ }^{1}$ \\ ${ }^{I}$ National ICT Australia, Victoria Research Laboratory, Dept. of Electricaland Electronic Engineering, University of Melbourne, Australia, \\ ${ }^{2}$ KTH Royal Institute of Technology, ICT School, Kista, Sweden \\ ${ }^{3}$ University of Zagreb, Faculty of Electrical Engineering and Computing, Dept. of Telecommunications, Croatia \\ ${ }^{4}$ Trinity College, Center for Telecommunication Value-Chain Research, Dublin, Ireland \\ sandua@student.unimelb.edu.au
}

\begin{abstract}
An approach to enhance core network survivability by utilizing dual-homing capabilities of the access network. Results reveal significant improvements in core network resource utilization and connection availability, suggesting benefits to network operators and service providers. OCIS codes: (060.0060) Fiber optics and optical communications; (060.4510) Optical communications
\end{abstract}

\section{Introduction}

Optical networks based on Wavelength Division Multiplexing (WDM) need to support very high data rates and therefore it is extremely important to ensure highly reliable trans mission with low outage times and data losses caused by failures. Optical networks can be divided into access and metro/core segments, where access network connects users directly to the Central Office while metro/core segment serves the aggregated traffic. Connections (i.e., lightpaths) provisioned over a metro/core network can typically recover from failures by switching from a failed working to a disjoint backup path. The backup resources can be pre-reserved at the connection setup time (protection) or dynamically provisioned after a failure occurs (restoration). In the access segment, one way to improve survivability is to provide protection for the shared part of the network, e.g., by dual-homing [1].

In a dual-homed optical access network architecture, a Local Exchange (LE) is connected to two different Metro/Core $(\mathrm{M} / \mathrm{C})$ nodes, protecting the access network against feeder fiber and/or M/C node failures. Such access network architecture is envisaged by the FP7 DISCUS project [2] and considered in this work. Fig. 1 shows the core network along with the dual-homed LEs. The core network comprises M/C nodes interconnected with an optical circuit switched wavelength layer. The $\mathrm{M} / \mathrm{C}$ node closest to each $\mathrm{LE}$ is considered as the primary home (denoted as S_pri_home for the source LE in Fig. 1), while the second closest node is considered as the secondary home (denoted as S_sec_home). Dual homing increases LE accessibility, thus enabling greater flexibility in establishing working and backup paths through the core network, i.e., using different combinations of primary and secondary homes (see Fig. 1) of source and destination LEs.

The benefits of dual-homing for core network survivability were investigated by the work of Wang et al.[1], where the authors proposed a coordinated protection scheme for dual-homing based IP-over-WDM networks where users (i.e., enterprises) connect to IP routers of different service providers. By considering dual homing only at the source of each communication request, the authors aimed at establishing a working and a backup path between both homes at the source side and the receiver at the destination, such that the total path length is minimized.

In this work, we investigate the benefits of utilizing dual-homing at all LEs for protection against core link failures. We as sume that the working path is always established between the two primary homes at each side, while the backup path can be established between any combination of the primary and secondary homes. We propose two h euristic algorithms to solve the Routing and Wavelength Assignment (RWA) problem with dedicated path protection for a static demand matrix such that: (i) the number of used wavelengths and (ii) the total path length is minimized. Due to the increased LE accessibility and a greater number of alternatives for protection paths, our proposed approach significantly reduces the average path length and the number of used wavelengths, and obtains higher connection availability compared to a baseline approach without dual homing in a country-wide Irish network.

\section{Problem Description}

For an end-to-end connection from the Source LE to the Destination LE, Fig. 1 illustrates the working path and possible backup paths in a dual-homed architecture. Considering this approach, we solve the RWA problem with dedicated path protection to enhance survivability of the core network. For each connection, we res erve resources for a pair of link-disjoint working and backup paths. As shown in Fig. 1, we assume that the working paths are always established between the primary homes of both source and destination LEs. However, thanks to dual-homing, the number of possible backup paths is higher providing more flexibility in how resources are used. The backup path can be chosen between any combination of primary and secondary homes at the source and destination side. 
The proposed RWA algorithm, called Dedicated Path Protection with Dual-Homing (DPP-DH), aims at providing survivability from core fiber failures in dual-homed networks. The physical topology of the network, i.e., the locations of M/C nodes, LEs, the set of physical links and their wavelength capacity, as well as the traffic matrix, are given as inputs to the algorithm. The traffic matrix between LE pairs is generated based on the user densities served by each LE. DPP-DH aims at establishing a pair of link-disjoint working and backup paths for each connection such that (i) the number of wavelengths or (ii) the total path length, is minimized. The details of the algorithm are provided in the next section.

\section{The Dedicated Path Protection with Dual-Homing Algorithm}

We design two variants of the DPP-DH algorithm with different optimization objectives. In the first variant, denoted as DPP-DH-W, the objective is to minimize the number of wavelengths used in the network. The second variant, denoted as DPP-DH-L, aims at minimizing the length of the established primary and backup paths allowing greater utilization of wavelengths. Algorithm 1 presents the pseudocode for DPP-DH-W.

In the beginning, traffic demands are sorted in the descending order of the product of their capacity and distance (CxD), thus making sure that the demands with higher capacity requirements over greater distances will be given priority when assigning resources. The first phase of the algorithm focuses on the selection of the working path. The sorted demands are considered one at a time. In steps 4 and 5, the shortest path between the primary homes of the source and destination LEs is computed on all of the wavelengths already in use, whose number is denoted as nActive_Wavelengths. The initial value of nActive_Wavelengths is 1 and it is incremented only if a working path cannot $\bar{b}$ e found on any of the already active wavelengths (step 11), in an effort to minimize the total number of wavelengths used. If all of the links on a computed shortest path do not have sufficient capacity to accommodate the current demand, the algorithm searches again (by returning to step 5) avoiding the most congested links of the path (steps 6-8), thus iteratively reaching the shortest path with available capacity. Once the shortest feasible path is

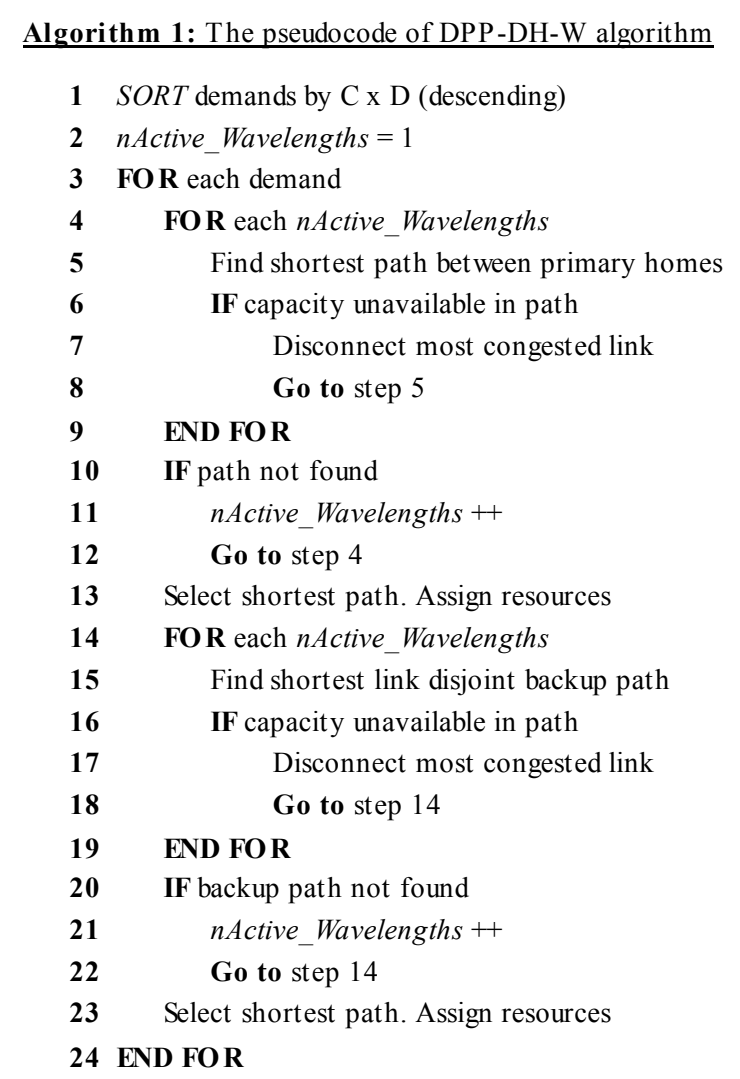

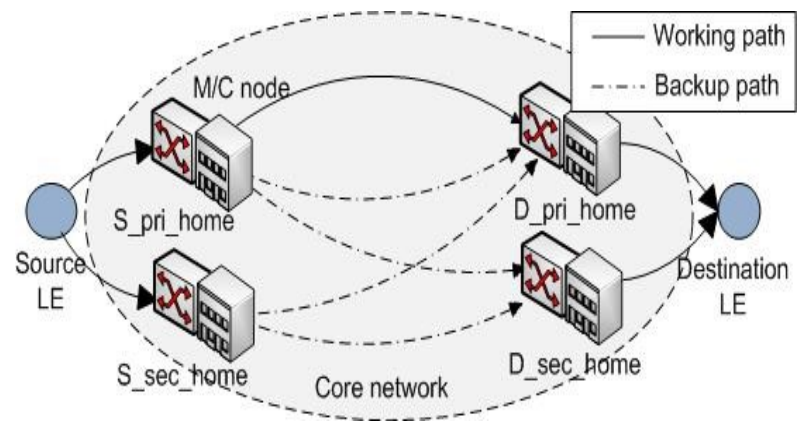

Fig. 1. The considered core network architecture with dual-homed Local Exchanges (LE) and different working/backup path options through the core.

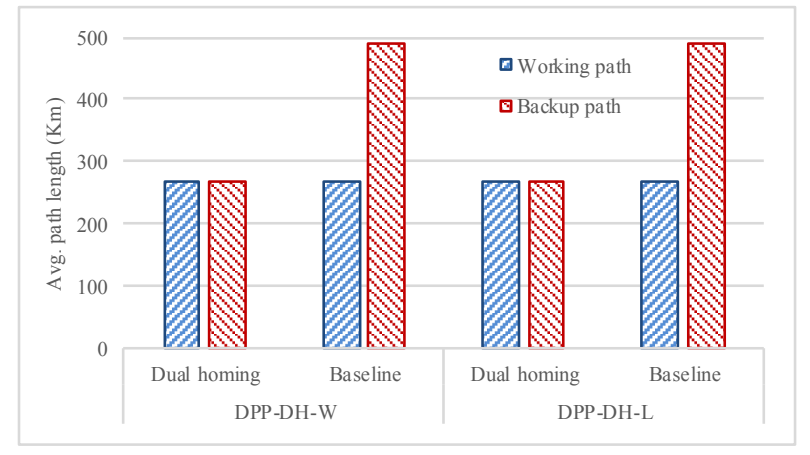

Fig. 2. Average path lengths for dual-homed and baseline architecture using DPP-DH-W and DPP-DH-L for $27 \mathrm{Tbit} / \mathrm{s}$ total net work traffic. 


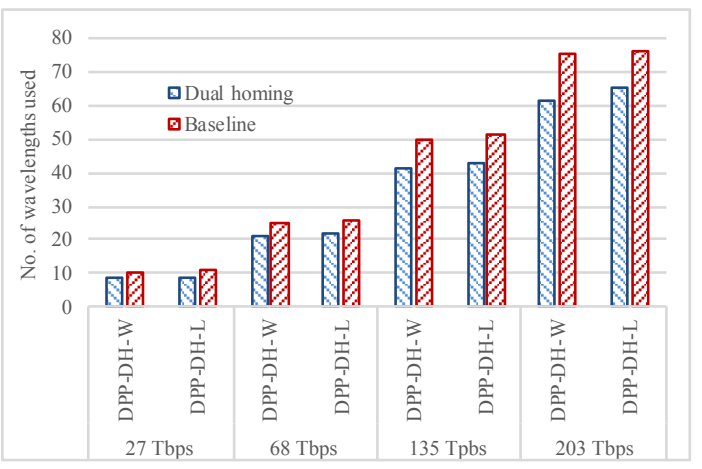

Fig. 3: The number of used wavelengths for different valuesof total network traffic load.

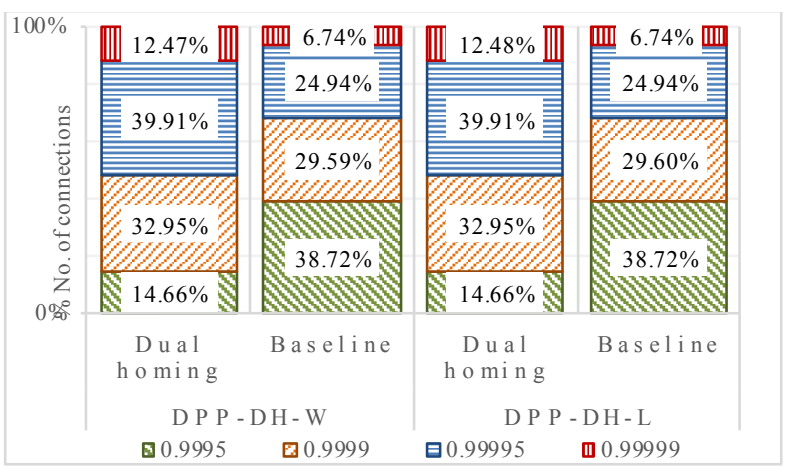

Fig. 4: The percentage of connections in different availabilitycategories for $27 \mathrm{Tbit} / \mathrm{s}$ total network traffic.

found for the current demand, network resources are reserved in step 13. Similarly, steps $14-24$ find and reserve network resources for a link-disjoint backup path. The DPP-DH-L algorithm is analogous to DPP-DH-W, with the difference of considering all available wavelengths in each step, i.e., without attempting to minimize their usage.

\section{Results and Discussion}

Both DPP-DH-W and DPP-DH-L algorithms were implemented and simulated in C\#. We used a countrywide network topology representing the Irish optical communication network, using a gravity model to generate traffic between all LE pairs. The traffic $\mathrm{T}_{\mathrm{AB}}$ between LEs A and B is calculated as: $T_{A B}=\left(\mathrm{K} \times C \times N_{A} \times N_{B}\right) / D^{2}$, where $\mathrm{K}$ is the traffic load factor, $C$ is the capacity provided per user, $N_{A}$ and $N_{B}$ represent the number of users served by LEs A and B respectively, and $D$ is the Euclidean distance between the two LEs. We consider fiber links supporting 80 wavelengths of $100 \mathrm{Gbit} / \mathrm{s}$ capacity and assume peak capacity of $10 \mathrm{Mbit} / \mathrm{s}$ per user. The generated traffic is scaled using different scaling factors $\mathrm{K}$ to model total network traffic intensities from $27 \mathrm{Tbit} / \mathrm{s}$ to $203 \mathrm{Tbit} / \mathrm{s}$. As a baseline scenario for evaluation purposes, we consider network architecture without dual homing. Therein, all working and backup pa ths are restricted to use the single (primary) $\mathrm{M} / \mathrm{C}$ node each $\mathrm{LE}$ is connected to.

Fig. 2 shows the average path length of the working and backup paths for the dual homing and baseline approaches when the total network load is $27 \mathrm{Tbit} / \mathrm{s}$. Results for higher network loads are analogous and omitted due to the space limitation. The results show that when dual homing is utilized for protection against fiber link failures, the backup path lengths are $45 \%$ smaller than in the baseline architecture with no dual homing. This is due to the higher number of options for the backup paths allowing for a possibly shorter backup path. Consequently, shorter backup paths result in a reduction of resource usage.

Fig. 3 shows the number of wavelengths used for different traffic loads in the network. In all cases, using DPPDH-W/-L in a network with dual homing results in a lower number of used wavelengths than in the baseline case. This is a very usefultrend as it reduces the overall cost for the network operators. Comparing the two variants ofDPP$\mathrm{DH}$, it is evident that DPP-DH-W uses fewer wavelengths than DPP-DH-L. This advantage is particularly visible in the scenarios with higher traffic load. Fig. 4 shows the percentages of connections satisfying different availability categories for $27 \mathrm{Tbit} / \mathrm{s}$ total traffic load. Both variants of DPP-DH obtain significant improvements in the percentage of connections with high availability $(99.999 \%$ and $99.995 \%)$, due to the path length reduction enabled by dual homing. Therefore, in general, DPP-DH achieves higher availability for connections whilst utilizing less network resources.

\section{Conclusion}

The paper studies the benefits of using dual-homing in access networks for providing resource-efficient protection from link failures in the core network. Two approaches for dedicated path protection with dual-homing, i.e.DPP-DH, are proposed. Compared to a baseline architecture without dual-homing, DPP-DH obtains a $45 \%$ reduction in the backup path length, an $18 \%$ reduction in wavelength usage, and an $84 \%$ and $60 \%$ increase in connections satisfying $99.999 \%$ and $99.995 \%$ availability, respectively.

Acknowledgement: This work is supported by the EC FP7, grant agreement No. 318137 DISCUS.

\section{References}

[1] J. Wang et al., J. Lightwave Technology., Vol. 23, No. 10, pp. 3111-3124, Oct. 2005.

[2] DISCUS Consortium, "Deliverable D2.1: Report on theinitial DISCUSEnd to End Architecture," 2013. 